\title{
Molecular Dynamics Simulation for Surface and Transport Properties of Fluorinated Silica Nanoparticles in Water or Decane: Application to Gas Recovery Enhancement
}

\author{
Kazem Sepehrinia* \\ School of Physics, Institute for Research in Fundamental Sciences, IPM, Farmanieh Building, \\ No. 70, Farmanieh Av., P.O. Box 19395-5531, Tehran - Iran \\ e-mail: physoffice@ipm.ir \\ * Corresponding author
}

\begin{abstract}
Determination of surface and transport properties of nanoparticles (NPS) is essential for a variety of applications in enhanced oil and gas recoveries. In this paper, the impact of the surface chemistry of silica NPs on their hydro- and oleo-phobic properties as well as their transport properties are investigated in water or decane using molecular dynamics simulation. Trifluoromethyl or pentafluoroethyl groups as water and oil repellents are placed on the NPs. It is found that the density and residence time of liquid molecules around the NPs are modulated considerably with the existence of the functional groups on the NPs'surfaces. Also, much larger density fluctuations for liquids close to the surface of the NPs are observed when the number of the groups on the NPs increases, indicating increased hydrophobicity. In addition, the diffusion coefficient of the NPs in either water or decane increases with increasing the number or length of the fluorocarbon chains, demonstrating nonBrownian behavior for the NPs. The surface chemistry imparts a considerable contribution on the diffusion coefficient of the NPs. Finally, potential of mean force calculations are undertaken. It is observed that the free energy of adsorption of the NPs on a mineral surface is more favorable than that of the aggregation of the NPs, which suggests the NPs adsorb preferably on the mineral surface.
\end{abstract}

Résumé - Simulation de la dynamique moléculaire pour les propriétés de surface et de transport des nanoparticules de silice fluorée dans l'eau ou le décane : Application à l'amélioration de la récupération des gaz — La détermination des propriétés de surface et de transport des nanoparticules est essentielle pour une variété d'applications dans les récupérations améliorées de pétrole et de gaz. Dans cet article, l'impact de la chimie de surface des nanoparticules de silice sur leurs propriétés hydro- et oleo-phobiques ainsi que leurs propriétés de transport sont étudiés dans l'eau ou le décane en utilisant la simulation de dynamique moléculaire. On place des groupes trifluorométhyle ou pentafluoroéthyle sous forme d'hydrofuges sur les nanoparticules. On constate que la densité et le temps de séjour des molécules liquides autour des nanoparticules sont modulés considérablement avec l'existence des groupes fonctionnels sur les surfaces des nanoparticules. En outre, des fluctuations de densité beaucoup plus grandes pour des liquides proches de la surface des nanoparticules sont observées lorsque le nombre des groupes sur les nanoparticules augmente, indiquant une hydrophobicité accrue. De plus, le coefficient de diffusion des nanoparticules dans l'eau ou le décane augmente avec l'augmentation du nombre ou de la longueur des chaînes 
fluorocarbonées, ce qui démontre un comportement non-Brownien pour les nanoparticules. La chimie de surface apporte une contribution considérable au coefficient de diffusion des nanoparticules. Enfin, les calculs de force moyenne sont effectués. On observe que l'énergie libre d'adsorption des nanoparticules sur une surface minérale est plus favorable que celle de l'agrégation des nanoparticules, ce qui suggère que les nanoparticules adsorbent de préférence sur la surface minérale.

\section{INTRODUCTION}

Nanoparticles (NPs) have found various applications in the oil and gas industry. Their applications as nanosensors [1], drilling fluid additives [2], and Enhanced Oil Recovery (EOR) agents [3-5] are among the main subjects of research. In EOR processes, NPs are used to alter reservoir rocks' wettability from oil-wet to water-wet in order to induce the immobile oil to flow, resulting in an increased oil recovery and, in turn, large economic benefits $[5,6]$. Similarly, in Enhanced Gas Recovery (EGR) processes, NPs are used to deliver a good level of oil and water repellency to the rock surface in order to alleviate oil and water blockage. For instance, in an experimental attempt, Mousavi et al. [7] proposed a method for EGR, by altering the wettability of reservoir rocks using fluorinated silica NPs. Adsorption of the fluorinated NPs to the inner walls of rock's porous media results in rough surfaces with a low free energy. These hydro- and oleo-phobic surfaces can markedly lead to the reduction of the capillary pressure, which mitigates liquid blockage, and, thus, the increase of recovery of gas reservoirs $[7,8]$.

There are a number of requirements to be considered in designing effective NPs for EGR applications. One of the requirements for NPs in order to remove liquid blockages is hydro- and oleo-phobicity. It has been demonstrated that macroscopic measures of hydro- and oleo-phobicity (e.g., contact angle measurements) do not extend to surfaces of NPs [9] and instead there are other measures for hydroand oleo-phobicity of nanomaterials such as superficial liquid-density profiles, liquid residence time, and local liquid-density fluctuations $[9,10]$. Another requirement for NPs in many of the applications is the ability to transport a desired distance within entrapped water and oil of porous media. Since diffusion controls the transport of NPs toward the interface between reservoir fluids and mineral surfaces [11], diffusion coefficient can be an enlightening property for probing transport of the chemical agents in reservoir fluids. The other requirement of such surface active agents is that they should not cause formation damage due to undesired plugging. Aggregated chemicals may block porous media of rock and reduce gas and condensate relative permeabilities [12].

Accordingly, a thorough understanding of surface and transport properties is required to design more effective functionalized NPs and overcome future obstacles in gas recovery. For this purpose, molecular simulation is envisioned to be an excellent tool, which is in the unique position of providing details that are lacking in experimental data.

In this vein, there have been molecular simulation studies reported on hydro- or oleo-phobic properties of NPs in dense fluids. For example, Koparde and Cummings [13] investigated interaction of water with $\mathrm{TiO}_{2}$ NPs. They reported that there is no significant increase in water density around the NPs, suggesting that the NPs are not hydrophilic. Accordino et al. [10] studied hydrophilic behavior of nanoscale grapheme-based materials by means of water density profiles and density fluctuations. They showed that lowering water-graphene attractive interactions (using simulation parameters) increases the water density fluctuations, indicating increased hydrophobicity. They confirmed that contrary to hydrophilic surfaces, changes for density profiles with pressure normal to the hydrophobic surfaces are appreciable. Jamadagni et al. [9] provided insights to the hydrophobicity of proteins and interfaces at nanoscale. They presented water density profiles and fluctuations close to the surfaces of self-assembled monolayers with solvent-exposed functions ranging from hydrophilic $\left(-\mathrm{OH}\right.$ and $\left.-\mathrm{CONH}_{2}\right)$ to hydrophobic $\left(-\mathrm{CH}_{3}\right.$ and $\left.-\mathrm{CF}_{3}\right)$. They found that fluctuations of water density near hydrophobic functions are larger. Fan et al. [14] investigated properties of silica NPs functionalized with methyl at the decane-water interface. They presented solvent density profiles and solvent residence autocorrelation functions near the NPs. Their results indicate that density profiles and residence autocorrelation functions for both decane and water are influenced by the nanoparticle surface properties.

Furthermore, Rudyak et al. [15] investigated the diffusion of NPs in dense fluids and concluded that the diffusion coefficients depend on the particle material. They have shown that the diffusion of NPs in dense fluids differs significantly from the diffusion of Brownian particles, governed by the Stokes-Einstein (SE) relation. Gao et al. [16] studied the effects of surface composition of hydrocarbon NPs on the diffusive behaviors of the NPs at/across water-benzene interfaces. They reported larger diffusion coefficients for hydrocarbon NPs than the corresponding values predicted using the SE relation. They also found that NPs with isotropic shape and uniform surface have smaller diffusion coefficients than those of NPs with comparable dimensions but anisotropic shapes and non-uniform surface composition. There are also other studies like [17, 18] 
that predict the SE relation for particle diffusion in nanoand molecular scales breaks down in a critical size of a few nanometers. These studies suggest that Molecular Dynamics (MD) simulation is more reliable approach than the SE relation for calculating diffusion coefficient of NPs.

In this paper, we provide insights into the behavior of wettability modifiers and present an approach for testing chemical agents before their extensive application in realistic conditions of gas reservoirs. MD simulations are used for investigating the properties of fluorinated silica NPs immersed in unbounded water or decane. In our simulations, decane represents oil phase of fluids in gas condensate reservoirs. Silica NPs functionalized with trifluoromethyl $\left(-\mathrm{CF}_{3}\right)$ or pentafluoroethyl $\left(-\mathrm{CF}_{2} \mathrm{CF}_{3}\right)$ groups are prepared and employed in our simulations for studying their hydroand oleo-phobic properties. Density profiles, residence time, and local liquid-density fluctuations are used to examine the impact of coverage percent and length of the fluorocarbon chains on the degree of hydro- and oleo-phobicity of NPs. Also, the relationship between hydro- and oleo-phobicity of the NPs and their diffusion coefficients is discussed. Finally, free energy of aggregation for two similar nanoparticles is compared with free energy of adsorption of them on $\alpha$-quartz 001 surface to ensure that the nanoparticles do not aggregate in clusters.

\section{METHODOLOGY}

In this section, we discuss simulation systems and details, used for studying properties of fluorinated silica NPs, and the force field employed to simulate the systems.

\subsection{Simulation Systems}

Simulated $\alpha$-quartz NPs were prepared in this manner: From the replicated unit cell of $\alpha$-quartz, a spherical cut of $3 \mathrm{~nm}$ in diameter was taken. To modify the surface chemistry, unsaturated silicon atoms were removed and the dangling oxygen atoms were terminated with one hydrogen atom [19]. According to experimental studies there is no $-\mathrm{Si}$ $(\mathrm{OH})_{3}$ groups on silica surface, thus, all $-\mathrm{Si}(\mathrm{OH})_{3}$ groups were deleted $[20,21]$. Functionalized NPs with different surface chemistry were built by tuning the number of $\mathrm{CF}_{3}$ or $-\mathrm{CF}_{2} \mathrm{CF}_{3}$ groups on their surface. A relaxation time of $100 \mathrm{ps}$ considered for NPs before using them in simulation systems. Seven NPs of different surface chemistry were studied. The NPs, shown in Figure 1, are: (1) hydroxylated silica nanoparticle, which is completely covered by $-\mathrm{OH}$ groups (HSNP); (2) nanoparticle covered by $25 \%-\mathrm{CF}_{3}$ groups (FSNP25); (3) nanoparticle covered by $55 \%$ $-\mathrm{CF}_{3}$ groups (FSNP55); (4) nanoparticle covered by

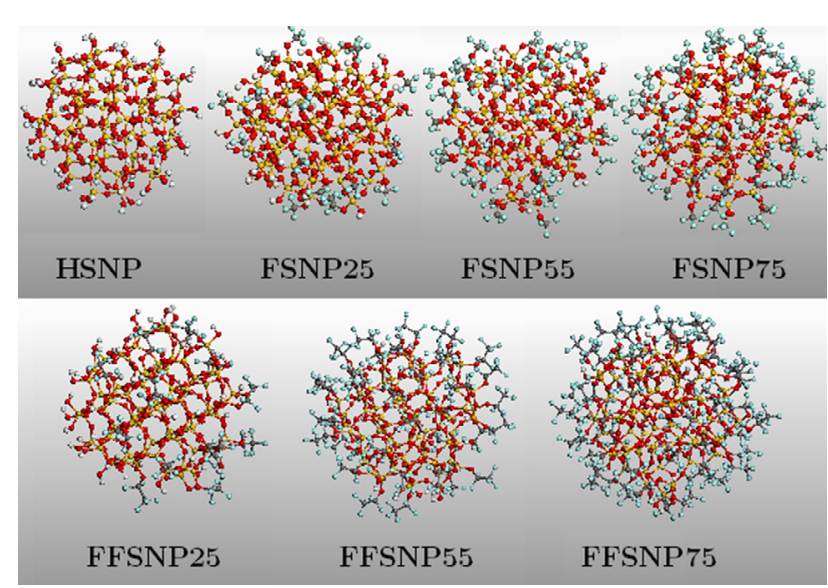

Figure 1

Simulated NPs. HSNP: hydroxylated silica nanoparticle; FSNP25: nanoparticle covered by $25 \%-\mathrm{CF}_{3}$ groups; FSNP55: nanoparticle covered by $55 \%-\mathrm{CF}_{3}$ groups; FSNP75: nanoparticle covered by $75 \%-\mathrm{CF}_{3}$ groups; FFSNP25: nanoparticle covered by $25 \%-\mathrm{CF}_{2} \mathrm{CF}_{3}$ groups; FFSNP55: nanoparticle covered by $55 \%-\mathrm{CF}_{2} \mathrm{CF}_{3}$ groups; FFSNP75: nanoparticle covered by $75 \%-\mathrm{CF}_{2} \mathrm{CF}_{3}$ groups. Atom colors: Silicon, yellow; Oxygen, red; Hydrogen, white; Carbon, gray; Fluorine, cyan.

$75 \%-\mathrm{CF}_{3}$ groups (FSNP75); (5) nanoparticle covered by $25 \%-\mathrm{CF}_{2} \mathrm{CF}_{3}$ groups (FFSNP25); (6) nanoparticle covered by $55 \%-\mathrm{CF}_{2} \mathrm{CF}_{3}$ groups (FFSNP55); (7) nanoparticle covered by $75 \%-\mathrm{CF}_{2} \mathrm{CF}_{3}$ groups (FFSNP75).

To examine properties of NPs in an unbounded liquid and calculating diffusion coefficients, a nanoparticle placed at the center of a cubic simulation cell with a side length of $70 \AA$. Combined structure was then created by inserting equilibrated liquid to remaining volume of the periodic box using wateradd subroutine of DL_POLY_CLASSIC. To avoid flying ice cube effect, the "no fic" subroutine of DL POLY was used in some limited intervals $(20 \mathrm{ps}$ in every $100 \mathrm{ps}$ ). This procedure was chosen by trial and error to avoid flying ice cube effect and also to have least possible effect of mass translation removal on diffusion dynamics. After that, a $4 \mathrm{~ns}$ MD simulation was run and the final states and trajectory of NPs in the system were used for analyzing the results. Figure 2 displays snapshots of simulation boxes for a nanoparticle in water and a nanoparticle in decane.

To perform Potential of Mean Force (PMF) calculations for adsorption of NPs, we used exactly the same $\alpha$-quartz slab and simulation cell of our former paper [22], which is shown in Figure 3.

\subsection{Simulation Details}

The initial structures of simulation systems were built using the ATEN [23]. MD simulations were carried out using 


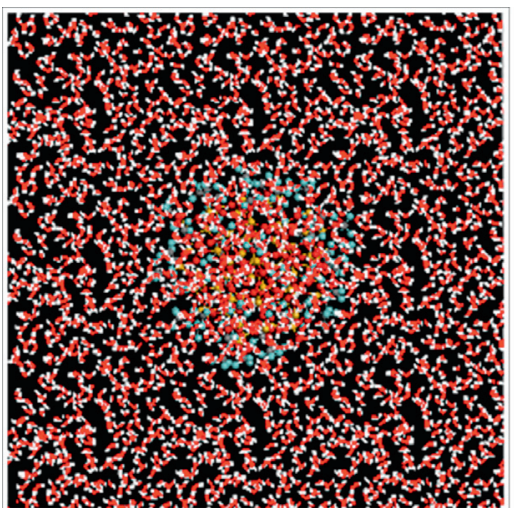

a)

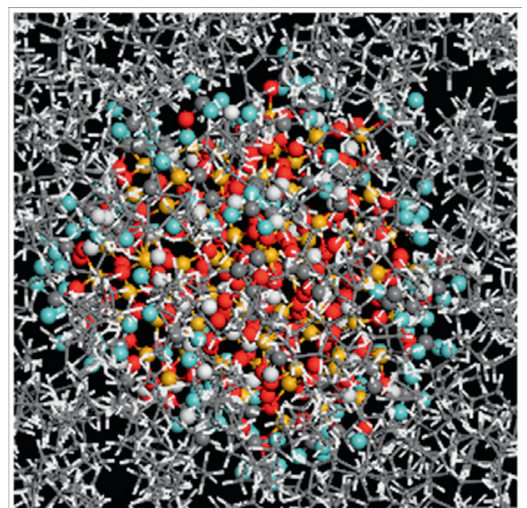

b)

Figure 2

Snapshot of simulation box. a) Initial configuration of FSNP55 in water and b) zoomed-in of FSNP55 in decane after 1 ns of production run. Atom colors: Silicon, yellow; Oxygen, red; Hydrogen, white; Carbon, gray; Fluorine, cyan.

the DL POLY CLASSIC [24] code. The long-range Coulomb interactions were handled using the Ewald summation technique to a precision of $10^{-5}$. A spherical atom based cutoff equal to $25 \AA$ was used. The velocityVerlet algorithm was used for integrating Newton's equations of motion with a time step of 1 fs. All simulations were carried out under the NVT ensemble using the Nosé-Hoover thermostat at $300 \mathrm{~K}$. Periodic boundary conditions were implemented along all three directions. Each run consisted of two periods; equilibration period and production period. Equilibration period was taken to be 500 ps. Production period was 4 ns.

\subsection{Force Field}

The force field proposed by Lopes et al. [25] is used for describing the intermolecular potential of functionalized $\alpha$-quartz NPs. This model was selected because it has been already used successfully for simulating $\alpha$-quartz-water [26] and $\alpha$-quartz-decane [27] interfaces. TIP3P [28] potential was employed to model water as it is compatible with the CHARMM force field. Also, CHARMM [29-31] force field was employed to model decane, and $-\mathrm{CF}_{3}$ and $-\mathrm{CF}_{2} \mathrm{CF}_{3}$ groups on the NPs. Hence, the functional form of the force field for all of the systems is as follows:

$$
\begin{aligned}
E_{\text {total }}= & \sum_{\text {bonds }} k_{r}\left(r-r_{0}\right)^{2}+\sum_{\text {angles }} k_{\theta}\left(\theta-\theta_{0}\right)^{2} \\
& +\sum_{\text {angles }} k_{\varphi}[1+\cos (n \varphi-y)]+\sum_{i<j} \varepsilon_{i j}\left[\left(\frac{R_{\min , i j}}{r_{i j}}\right)^{12}\right. \\
& \left.-\left(\frac{R_{\min , i j}}{r_{i j}}\right)\right]^{6}+\sum_{i<j} k \frac{q_{i} q_{j}}{r_{i j}}
\end{aligned}
$$

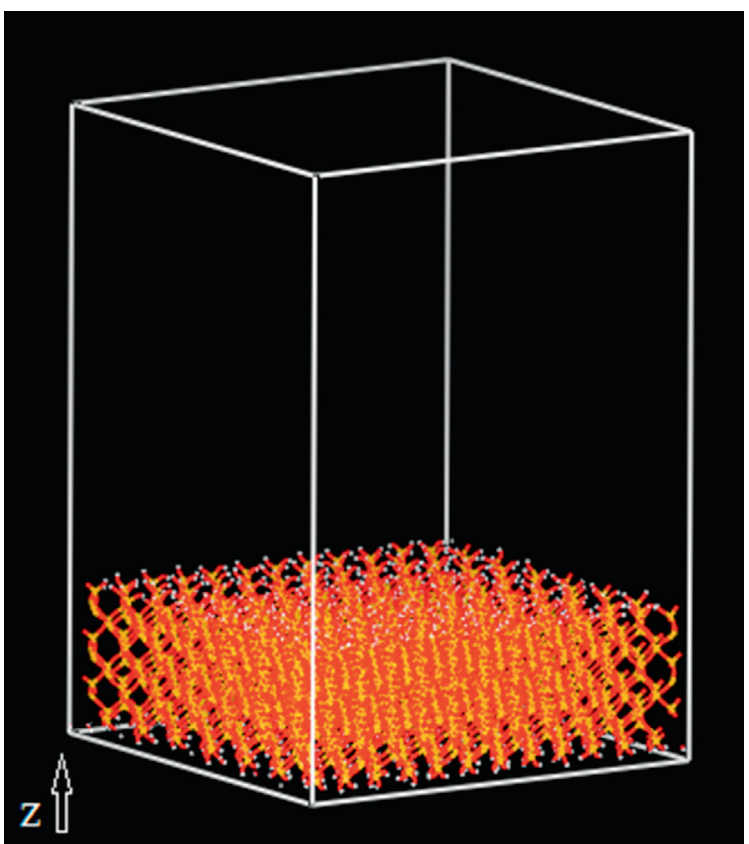

Figure 3

Simulation cell containing only hydroxylated $\alpha$-quartz slab with a vacuum gap in the $z$ direction, used for PMF calculations. Atom colors: Silicon, yellow; Oxygen, red; Hydrogen, white.

where the first three sums are over bonded interactions (bonds, angles and dihedrals), and the last two sums are over non-bonded interactions (van der Waals and electrostatic). In addition, the Lennard-Jones parameters for unlike atoms are obtained from the Lorentz-Berthelot combining rule, i.e., arithmetic and geometric mean rules for the cross Lennard-Jones size parameter and the cross Lennard-Jones energy parameter, respectively. All of the force field 
TABLE 1

Non-bonded parameters for the components of our simulation systems.

\begin{tabular}{c|c|c|c|c}
\hline Atom key & Remark & $\varepsilon(\mathrm{kcal} / \mathrm{mol})$ & $R_{\min } / 2(\AA)$ & 2.2000 \\
\hline $\mathrm{Si}$ & Bulk silicon & -0.6000 & 1.7700 & 1.0800 \\
\hline $\mathrm{OSiE}$ & Bulk oxygen & -0.1521 & 1.7700 & -0.5425 \\
\hline $\mathrm{OSiH}$ & Hydroxyl surface oxygen & -0.1521 & 0.2245 & 0.5400 \\
\hline $\mathrm{HSiO}$ & Hydroxyl surface hydrogen & -0.0460 & 2.1750 & 0.6020 \\
\hline $\mathrm{C}$ & Carbon of fluorine chain & -0.0550 & 1.6300 & -0.1450 \\
\hline $\mathrm{F}$ & Fluorine of fluorine chain & -0.1350 & 1.7682 & -0.8340 \\
\hline $\mathrm{OT}$ & Water oxygen & -0.1521 & 0.2245 & 0.4170 \\
\hline $\mathrm{HT}$ & Water hydrogen & -0.0460 & 2.0100 & -0.1800 \\
\hline $\mathrm{CM}$ & Middle carbon of decane & -0.0560 & 2.0500 & -0.2710 \\
\hline $\mathrm{CE}$ & Ending carbon of decane & -0.0780 & 1.3400 & 0.0900 \\
\hline $\mathrm{HM}$ & Middle hydrogen of decane & -0.0350 & 1.3400 & 0.0900 \\
\hline
\end{tabular}

TABLE 2

Bonded parameters for the components of our simulation systems: bonds.

\begin{tabular}{c|c|c}
\hline Bond & $k_{r}\left(\mathrm{kcal} / \mathrm{mol} . \AA^{2}\right)$ & $r_{0}(\AA)$ \\
\hline Si-OSiE & 302.0000 & 1.6980 \\
\hline Si-OSiH & 325.0000 & 1.6800 \\
\hline OSiH-HSiO & 566.0000 & 0.9750 \\
\hline OSiH-C & 363.2140 & 1.4150 \\
\hline C-F & 432.5890 & 1.3400 \\
\hline $\mathrm{C}-\mathrm{C}$ & 224.3212 & 1.5250 \\
\hline OT-HT & 450.0000 & 0.9572 \\
\hline CM-CE & 222.5000 & 1.5300 \\
\hline CM-CM & 222.5000 & 1.5280 \\
\hline CM-HM & 309.0000 & 1.1110 \\
\hline CE-HE & 322.0000 & 1.1110 \\
\hline
\end{tabular}

parameters for components of the system are given in Tables 1-4. These tables are updated due to presence of $\mathrm{CF}_{2} \mathrm{CF}_{3}$ groups on the NPs.

\section{RESULTS AND DISCUSSION}

In the following sections we present our results and discussions. We examine the general features of the surface and transport properties of our NPs by dividing them into two classes, those functionalized with $-\mathrm{CF}_{3}$ groups (the first set), and those functionalized with $-\mathrm{CF}_{2} \mathrm{CF}_{3}$ groups (the second set). This classification helps us to understand the impact of the length of $-\mathrm{CF}_{3}$ and $-\mathrm{CF}_{2} \mathrm{CF}_{3}$ groups. We first consider radial density profiles of liquids around the NPs (Sect. 2.1), and local liquid-density fluctuations close to the surface of the NPs (Sect. 2.2). Then residence time of liquid molecules (or atoms) in a spherical shell next to the surface of the NPs are calculated and reported (Sect. 2.3), and diffusion coefficients of the NPs are presented (Sect. 2.4). Finally, PMF calculations for aggregation and adsorption of the NPs on the $\alpha$-quartz 001 surface are given in Section 2.5.

\subsection{Density Profiles}

In this section, the radial density profiles of $\mathrm{O}_{\mathrm{w}}$ (oxygen of water) and $\mathrm{C}_{\mathrm{d}}$ (carbon of decane) are reported as a function of the distance from the surface silicon atoms of the NPs. In our results, each density plot comes with the corresponding density profile for HSNP, as a reference for measuring the degree of hydro- and oleo-phobicity of our NPs.

The radial density profiles of $\mathrm{O}_{\mathrm{w}}$ for the first set, embedded in unbounded water, are shown in Figure 4a. Qualitative shape of the density profile for HSNP and the bulk number density of 0.033 agree well with previously reported MD simulations $[14,32]$. A short-ranged ordering for water 
TABLE 3

Bonded parameters for the components of our simulation systems: angles.

\begin{tabular}{|c|c|c|}
\hline Angles & $k_{\theta}\left(\mathrm{kcal} / \mathrm{mol} \mathrm{rad}^{2}\right)$ & $\theta_{0}\left({ }^{\circ}\right)$ \\
\hline OSiE-Si-OSiE & 30.0000 & 117.0000 \\
\hline $\mathrm{Si}-\mathrm{OSiE}-\mathrm{Si}$ & 34.0000 & 150.0000 \\
\hline $\mathrm{Si}-\mathrm{OSiH}-\mathrm{HSiO}$ & 34.0000 & 122.5000 \\
\hline OSiE-Si-OSiH & 32.0000 & 126.0000 \\
\hline $\mathrm{OSiH}-\mathrm{Si}-\mathrm{OSiH}$ & 30.0000 & 117.0000 \\
\hline $\mathrm{Si}-\mathrm{OSiH}-\mathrm{C}$ & 78.6580 & 114.9430 \\
\hline $\mathrm{OSiH}-\mathrm{C}-\mathrm{C}$ & 105.0000 & 112.8000 \\
\hline $\mathrm{OSiH}-\mathrm{C}-\mathrm{F}$ & 114.6410 & 106.9000 \\
\hline $\mathrm{C}-\mathrm{C}-\mathrm{F}$ & 108.2500 & 111.2010 \\
\hline $\mathrm{F}-\mathrm{C}-\mathrm{F}$ & 117.8800 & 106.0810 \\
\hline HT-OT-HT & 55.0000 & 104.5200 \\
\hline $\mathrm{CM}-\mathrm{CM}-\mathrm{CM}$ & 58.3500 & 113.6000 \\
\hline $\mathrm{CM}-\mathrm{CM}-\mathrm{CE}$ & 58.0000 & 115.0000 \\
\hline $\mathrm{CM}-\mathrm{CM}-\mathrm{HM}$ & 26.5000 & 110.1000 \\
\hline CE-CM-HM & 34.6000 & 110.1000 \\
\hline $\mathrm{HM}-\mathrm{CM}-\mathrm{HM}$ & 35.5000 & 109.0000 \\
\hline CM-CE-HE & 34.6000 & 110.1000 \\
\hline $\mathrm{HE}-\mathrm{CE}-\mathrm{HE}$ & 35.5000 & 108.4000 \\
\hline
\end{tabular}

molecules is observed from the density profiles. The magnitude of the ordering is much less than that typically observed for flat mineral surfaces [33]. This fact, probably, arises from the sphericity and roughness of the NPs' surfaces, which prevents the formation of layers with higher densities. However, with increasing the number of $-\mathrm{CF}_{3}$ groups, water molecules are depleted slightly from the hydration shell next to the surface of the NPs.

The radial density profiles of $\mathrm{O}_{\mathrm{w}}$ around the second set of the nanoparticles are shown in Figure 4b. A layer next to the surface of the NPs, with the density lower than that of the bulk, is observable in the profiles. With increasing the number of $-\mathrm{CF}_{2} \mathrm{CF}_{3}$ groups, water molecules are depleted considerably from the hydration shell next to the surface of the NPs. The second set of our NPs represents stronger water-repellency behavior compared to the first set, suggesting that larger fluorocarbon chains increase greatly the hydrophobicity of our NPs.

In the density profiles of $\mathrm{C}_{\mathrm{d}}$ for the first set of the NPs (Fig. 5a), a considerable ordering is observed, probably because the decane molecule is larger and cannot fit the
TABLE 4

Bonded parameters for the components of our simulation systems: dihedrals.

\begin{tabular}{c|c|c|c}
\hline Dihedral & $\begin{array}{c}k_{\varphi} \\
(\mathrm{kcal} / \mathrm{mol})\end{array}$ & $n$ & $\gamma\left({ }^{\circ}\right)$ \\
\hline OSiE-Si-OSiE-Si & 0.1800 & 5 & 0.0000 \\
\hline OSiE-Si-OSiH-HSiO & 0.3000 & 3 & 0.0000 \\
\hline OSiH-Si-OSiH-HSiO & 0.3500 & 3 & 0.0000 \\
\hline Si-OSiE-Si-OSiH & 0.1800 & 5 & 0.0000 \\
\hline OSiE-Si-OSiH-C & 0.0750 & 3 & 0.0000 \\
\hline OSiH-Si-OSiH-C & 0.0750 & 3 & 0.0000 \\
\hline Si-OSiH-C-F & 0.1000 & 3 & 0.0000 \\
\hline OSiH-C-C-F & 0.3150 & 3 & 0.0000 \\
\hline F-C-C-F & 0.3500 & 3 & 0.0000 \\
\hline CM-CM-CM-CM & 0.0645 & 2 & 0.0000 \\
\hline CM-CM-CM-CE & 0.2039 & 5 & 0.0000 \\
\hline CM-CM-CM-HM & 0.1950 & 3 & 0.0000 \\
\hline CE-CM-CM-HM & 0.1800 & 3 & 0.0000 \\
\hline HM-CM-CM-HM & 0.2200 & 3 & 0.0000 \\
\hline CM-CM-CE-HE & 0.1600 & 3 & 0.0000 \\
\hline HM-CM-CE-HE & 0.1600 & 3 & 0.0000 \\
\hline
\end{tabular}

atomic-scale roughness present at the nanoparticle's surfaces. The density profiles are not modulated considerably by increasing the number of $-\mathrm{CF}_{3}$ groups, demonstrating the weakness of the first set of our fluorinated silica NPs in oil repellency. This result is in agreement with experimental studies, concluding that the ability of oil repellency for fluorinated surface-active agents is less than their ability of water repellency [7, 34, 35]. In our previous study [22] we highlighted that to obtain more general and reliable conclusions regarding the effects of fluorinated surfaceactive agents on the oil repellency, larger fluorocarbon chains on the NPs, such as $-\mathrm{CF}_{2} \mathrm{CF}_{3}$, must be used. Herein we have investigated the impact of such fluorocarbon chains. Results for the second set of the NPs are interesting. Stronger oil repellency is observed in Figure 5b. First peak position for each of the functionalized NPs is shifted at least $1 \AA$ away from the surface and the maximum density of $C_{d}$ at the peaks is almost the same as the bulk number density (0.0217). Ostensibly, longer chains of fluorocarbon, in addition to high surface activity, provide smaller space for decane molecules to penetrate and wet the surface of the NPs. 


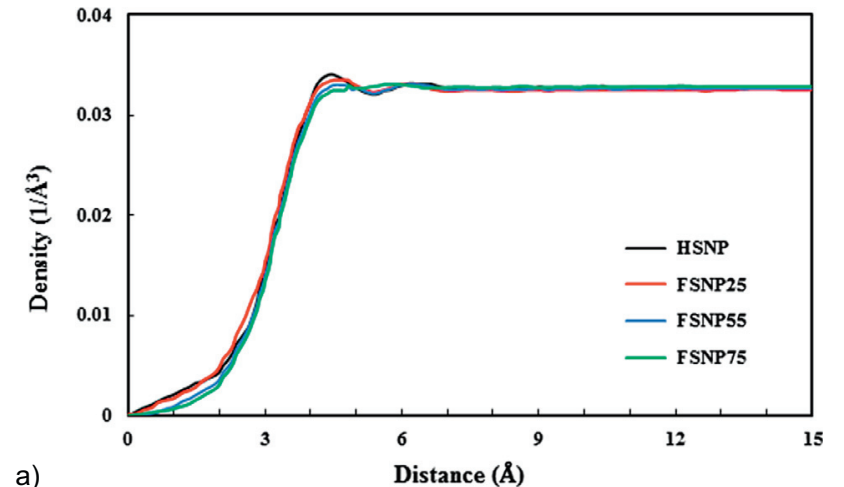

a)

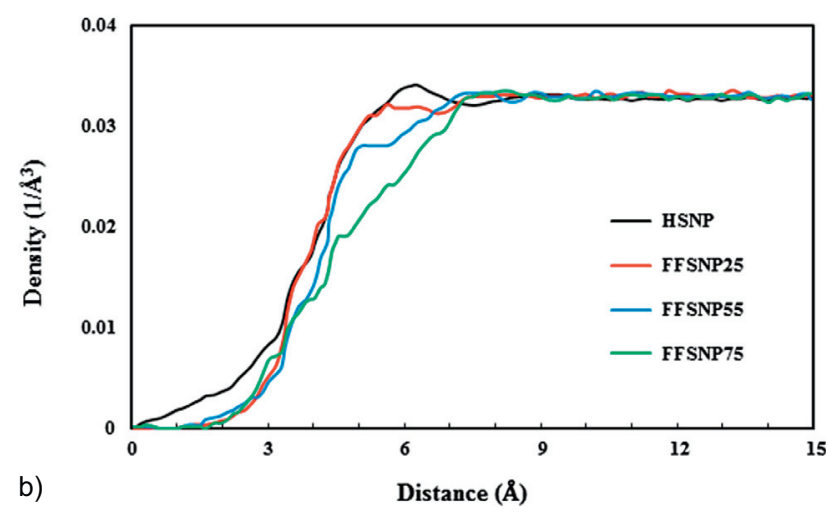

Figure 4

The radial number-density profiles of $\mathrm{O}_{\mathrm{w}}$ around the NPs as a function of the distance from the nanoparticle surface. $(a, b)$ The density profiles of $\mathrm{O}_{\mathrm{w}}$ around the first and second sets of the NPs. The distance from the nanoparticle surface (at $z=0$ ) is defined as the time-averaged position of all surface silicon atoms.

\subsection{Density Fluctuations}

An appropriate measure of surface hydro- or oleo-phobicity is local liquid-density fluctuation. This quantity measures local hydro- or oleo-phobicity, and is related to liquid liability or removal/vacating probability. It has been revealed that superficial liquid-density profiles do not well-indicate surface hydro- or oleo-phobicity [36]. This can be explained by the fact that liquid-density profiles, which account for ordering of liquid molecules, demonstrate similar oscillatory behavior in the vicinity of different surfaces, and display no apparent correlation with macroscopic observations, such as contact angle measurements. However, contrary to hydro- or oleo-philic surfaces, which are subject to strong attractive interactions with liquid molecules, interactions are weaker at hydro- or oleo-phobic surfaces, and liquid molecules display low residence times at hydro- or oleo-phobic surfaces. Thus, such layers display enhanced density fluctuations [9]. In particular, liquids at hydro- or oleo-phobic surfaces do in fact present much larger density fluctuation
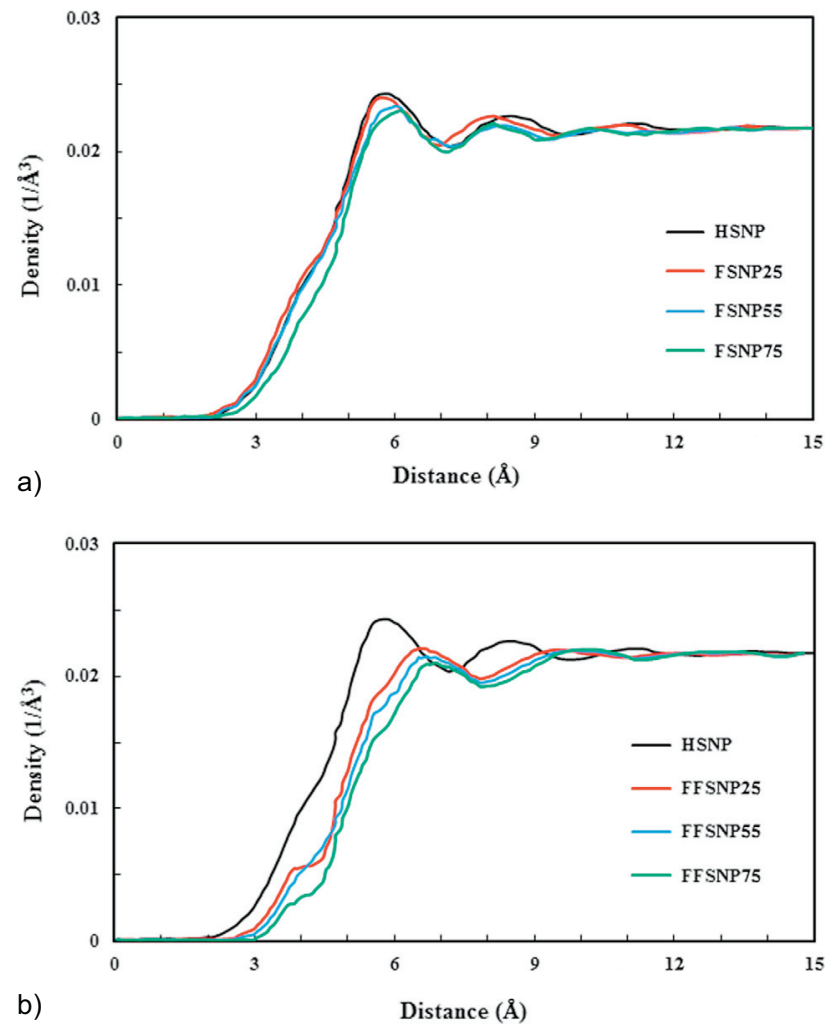

Figure 5

The radial number-density profiles of $\mathrm{C}_{\mathrm{d}}$ around the NPs as a function of the distance from the nanoparticle surface. $(\mathrm{a}, \mathrm{b})$ The density profiles of $\mathrm{C}_{\mathrm{d}}$ around the first and second sets of the NPs. The distance from the nanoparticle surface (at $z=0$ ) is defined as the time-averaged position of all surface silicon atoms.

than the ones displayed by hydro- or oleo-philic surfaces, thus providing an appropriate quantitative measure for hydro- or oleo-phobicity.

The normalized fluctuation of liquid number-density within an observation volume is defined mathematically as:

$$
\sigma_{N}^{2} /\langle N\rangle^{2}
$$

where $N$ is the number of liquid molecules within the volume, $\sigma_{N}$ the standard deviation in $N$, and $\langle N\rangle$ the average of $N$ in the simulation run. The fluctuation is approximately equal to $k_{\mathrm{B}} T /\left(2 \mu^{\mathrm{ex}}\right)$, with $\mu^{\mathrm{ex}}$ being the free energy of formation of the volume, representing that a high value of the normalized density fluctuation at a given position indicates a favorable work of volume creation at such position and, thus, a high hydro- or oleo-phobicity [10].

Here, theoretical expectation of enhanced liquid-density fluctuations near hydro- and oleo-phobic NPs is borne out quantitatively with the aid of our simulation data. Since 
NPs are not homogeneous we calculated number of liquid molecules (number of $\mathrm{C}_{\mathrm{d}}$ atoms in the case of decane) in a spherical shell with the thicknesses of 2, 3 and $4 \AA$ next to the NPs' surfaces. Figure 6 shows the values of $\sigma_{N}^{2} /\langle N\rangle^{2}$ for liquid molecules in the spherical shell observation volume of thickness $2 \AA$ for NPs. Larger fluctuations are observed with increasing the number of $-\mathrm{CF}_{3}$ groups for the first set of NPs. This is also the case for the NPs functionalized with pentafluoroethyl except that fluctuations are larger than those for the first set of NPs. Comparing results for the two sets indicates that, in addition to the number of functional groups, larger fluorocarbon chains on the NPs exhibit considerably improved oleophobicity. Higher liability of water or decane molecules near the fluorinated NPs indicates increased hydro- and oleo-phobicity of fluorinated NPs.

The normalized fluctuations of liquid number-density for water and decane in the three observation volumes are compared in Table 5. Shown in the table is the following quantity:

$$
\phi_{\mathrm{wd}}=\frac{\sigma_{N}^{2} /\langle N\rangle^{2} \text { for water }}{\sigma_{N}^{2} /\langle N\rangle^{2} \text { for decane }}
$$

which quantifies the ratio of the normalized fluctuation of water number-density to the corresponding value for decane in a simulation run. There are no significant differences in values of $\phi_{\mathrm{wd}}$ for different observation volumes. The results show that $\phi_{\mathrm{wd}}$ is always greater than 1 , and fluctuations for water molecules are larger than those for decane molecules. This indicates weakness of our NPs in oil repellency compared with their water repellency. Since most of fluorinated materials employed to alternate the wettability are considerably hydrophobic, ratios closer to 1 indicates that the material has also considerable oleophobicity.

\subsection{Residence Time}

To explain further the interaction of NPs with solvent molecules, residence time of solvents in the first hydration shell of NPs was calculated. We used trajectory of our molecular dynamics simulations for calculating residence time correlation function from Equation (2) [37]:

$$
\langle R(t)\rangle=\left\langle\frac{1}{N_{0}} \sum_{i=1}^{N_{t}} \theta_{i}(0) \theta_{i}(t)\right\rangle
$$

where $N$ is the number of liquid molecules (or number of a specific atom of a molecule) within the first hydration layer from the surface and $\theta_{i}$ is the Heaviside function, which is

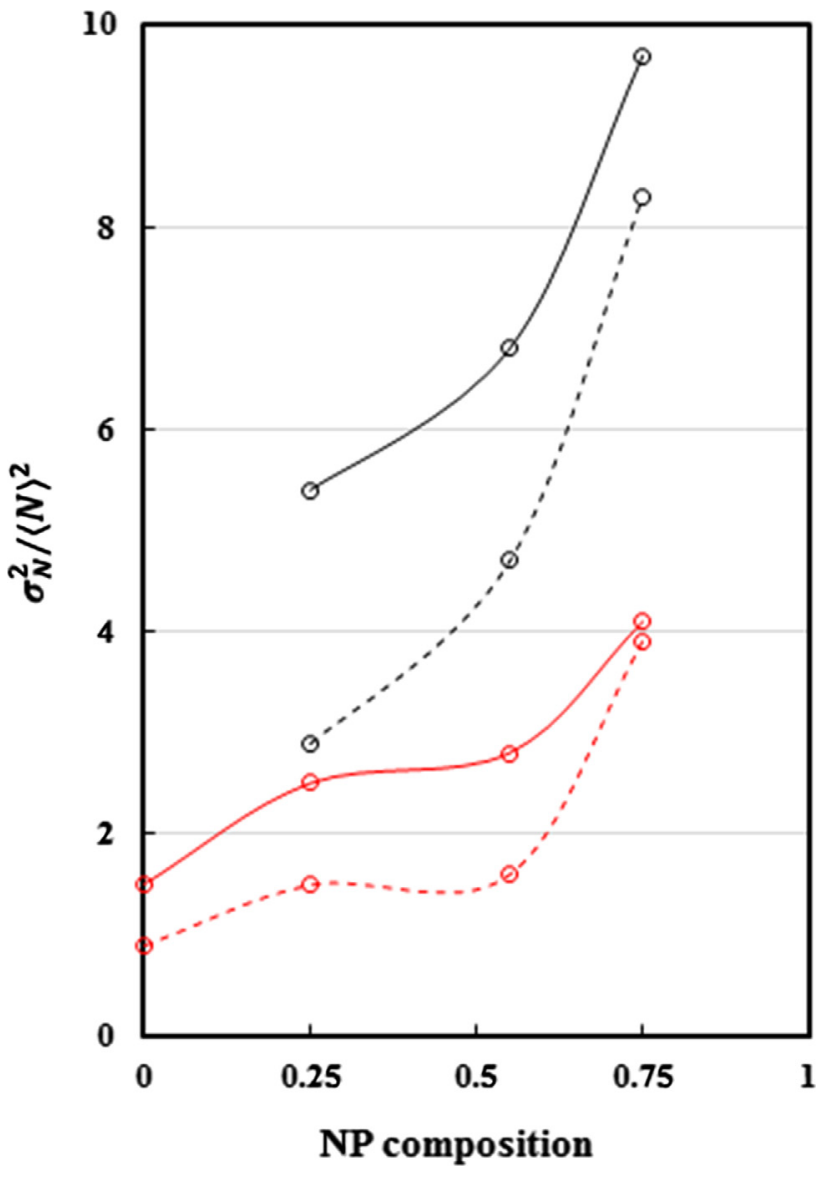

Figure 6

Water (solid line) and decane (dashed line) density fluctuations in a spherical shell with the thickness of $2 \AA$ next to the surface of the first (red lines) and second (black lines) sets of NPs in the simulation run.

1 when the molecule (or atom) $i$ lies inside the spherical shell of first hydration layer and 0 otherwise. Then the residence time will be determined as follows:

$$
\tau=\int_{0}^{\infty}\langle R(t)\rangle \mathrm{d} t
$$

Residence times for water and carbon of decane in the first hydration shell of our NPs are presented in Table 6. Residence times for water and carbon of decane decrease rapidly as the number of $-\mathrm{CF}_{3}$ or $-\mathrm{CF}_{2} \mathrm{CF}_{3}$ groups on the NPs increases. Residence times for the second set of the NPs are less than those obtained for the first set of the NPs, except that going from FSNP75 to FFSNP25 there is increase in residence time both for water and decane. This means that coverage percent is more important factor than length of fluorine chain in measuring degree of hydroand oleo-phobicity of NPs. Residence time is an indicator 
TABLE 5

Comparison of $\phi_{\mathrm{wd}}$ for different NPs in a simulation run.

\begin{tabular}{c|c|c|c|c|c|c|c}
\hline NPs & HSNP & FSNP25 & FSNP55 & FSNP75 & FFSNP25 & FFSNP55 & FFSNP75 \\
\hline$\phi_{\mathrm{wd}}$ for 2 $\AA$ observation volume & 1.66 & 1.66 & 1.75 & 1.05 & 1.86 & 1.44 & 1.16 \\
\hline$\phi_{\mathrm{wd}}$ for 3 $\AA$ observation volume & 1.64 & 1.61 & 1.68 & 1.03 & 1.83 & 1.37 & 1.22 \\
\hline$\phi_{\mathrm{wd}}$ for 4 $\AA$ observation volume & 1.6 & 1.59 & 1.71 & 1.09 & 1.84 & 1.46 & 1.15 \\
\hline
\end{tabular}

TABLE 6

Residence time (ps) for water and carbon of decane in the first hydration shell of NPs.

\begin{tabular}{|c|c|c|c|c|c|c|c|}
\hline NPs & HSNP & FSNP25 & FSNP55 & FSNP75 & FFSNP25 & FFSNP55 & FFSNP75 \\
\hline Residence time of water & $4.3 \pm 0.2$ & $3.2 \pm 0.1$ & $3.1 \pm 0.15$ & $2.7 \pm 0.1$ & $2.9 \pm 0.1$ & $2.2 \pm 0.11$ & $1.9 \pm 0.1$ \\
\hline Residence time of $C_{d}$ & $5.6 \pm 0.2$ & $4.5 \pm 0.1$ & $4.4 \pm 0.1$ & $3.8 \pm 0.1$ & $4.1 \pm 0.12$ & $3.2 \pm 0.1$ & $2.5 \pm 0.1$ \\
\hline
\end{tabular}

TABLE 7

Diffusion coefficient $\left(10^{-6} \mathrm{~cm}^{2} / \mathrm{s}\right)$ of NPs in liquid water or decane.

\begin{tabular}{c|c|c|c|c|c|c|c}
\hline NPs & HSNP & FSNP25 & FSNP55 & FSNP75 & FFSNP25 & FFSNP55 & FFSNP75 \\
\hline Diffusion coefficient in water & $1.53 \pm 0.07$ & $3.1 \pm 0.1$ & $5.68 \pm 0.1$ & $5.72 \pm 0.16$ & $4.8 \pm 0.09$ & $5.9 \pm 0.13$ & $7.39 \pm 0.2$ \\
\hline Diffusion coefficient in decane & $1.61 \pm 0.09$ & $2.5 \pm 0.1$ & $3.4 \pm 0.11$ & $4.8 \pm 0.12$ & $3.2 \pm 0.1$ & $4.5 \pm 0.14$ & $6.7 \pm 0.18$ \\
\hline
\end{tabular}

of extent of binding of a liquid to a surface and it has decreased considerably for functionalized NPs. As it shall be discussed later in this paper, this fact eases diffusion of functionalized NPs across the bulk liquid.

\subsection{Diffusion Coefficients}

Diffusion coefficient is related closely to hydro- and oleophobicity of NPs. When a nanoparticle is highly hydro- or oleo-phobic, extent of binding of liquid molecules to its surface decreases and the nanoparticle diffuses faster across the liquid. As an explanation of this behavior, let us look at spherical hydro- or oleo-philic surfaces. H-bonding network and layered structure of liquid molecules around the NPs results in a dense solvation shell, which leads to a larger effective radius of the nanoparticle, and thus, according to the SE equation, a reduced diffusion coefficient. We examine the certainty of this prediction for fluorinated silica NPs.

During MD simulations, the velocities of molecules and NPs at sequential times are calculated. From these variables, using methods of non-equilibrium statistical mechanics it is possible to determine all thermodynamic and transport characteristics of the system. In particular, the self-diffusion coefficient $D$ is determined using the Green-Kubo formula [15, 38]:

$$
D=\int_{0}^{\mathrm{T}} \chi(t) \mathrm{d} t
$$

where $\chi(t)=\langle V(0) \cdot V(t)\rangle$ is the Velocity AutoCorrelation Function (VACF). Here $V$ is the nanoparticle velocity and the angular brackets denote averaging over the number of runs. $T$ is the time when the VACF reaches the plateau value, i.e., the time for which the value of diffusion coefficient stops to noticeably vary within the accuracy of calculations [39].

From the classical theory, for a spherical particle of radius $a$ in a liquid of viscosity $\eta$, the diffusion coefficient can be estimated using the SE relation:

$$
D=\frac{k_{\mathrm{B}} T}{6 \pi \eta a}
$$

where $k_{\mathrm{B}}$ is the Boltzmann constant, and $T$ the absolute temperature. For determining the diffusivity of NPs through the SE relation, the viscosity of the liquid is calculated without the NPs in the system. In this vein, 


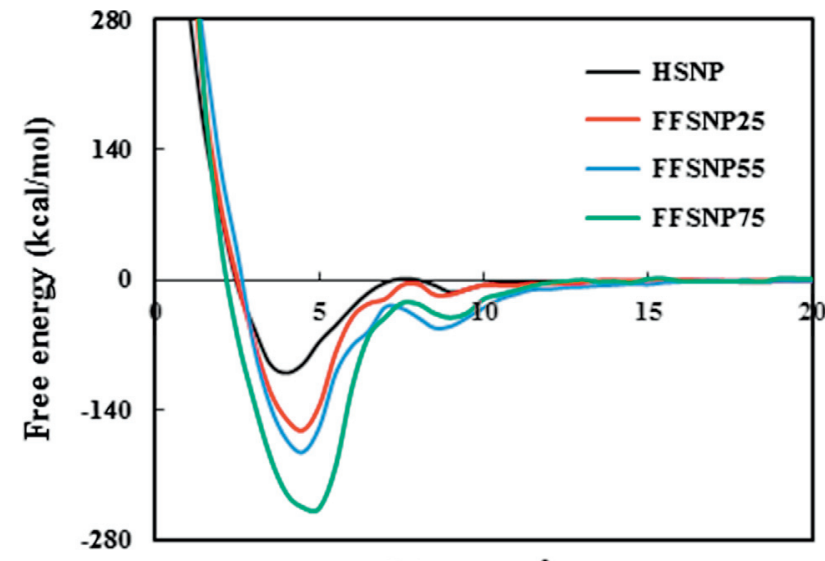

a)

Distance ( $(\mathbf{\AA})$

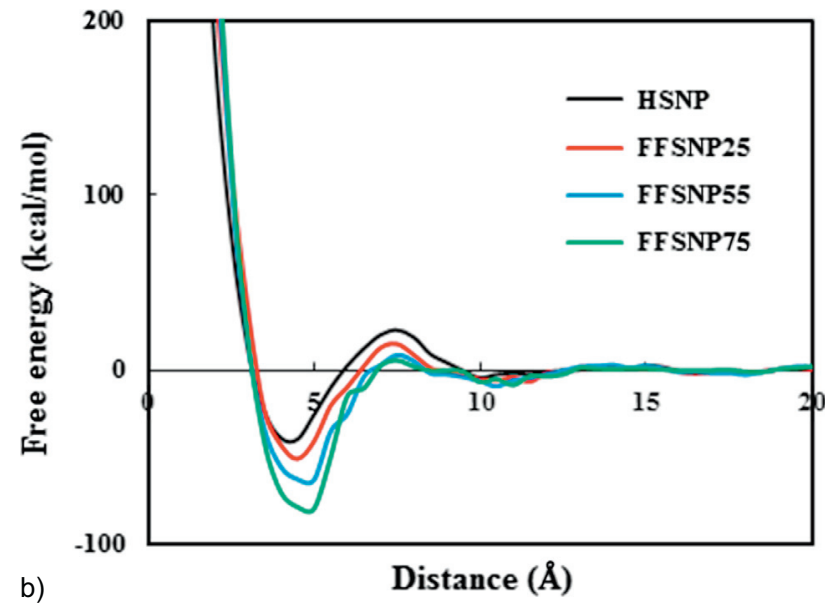

Figure 7

The free energy calculated for the NPs functionalized with $-\mathrm{CF}_{2} \mathrm{CF}_{3}$ groups in a water-loaded pore. (a, b) The free energy of adsorption on hydroxylated $\alpha$-quartz surface and the free energy of aggregation for the NPs.

we conducted independent simulations in a rectangular cell and applied the Green-Kubo formalism to calculate the viscosity using [40]:

$$
\eta=\frac{V}{k_{\mathrm{B}} T} \int_{0}^{\infty}\left\langle P_{x y}(0) P_{x y}(t)\right\rangle \mathrm{d} t
$$

where $V$ is the volume of rectangular cell, angular brackets denote ensemble averaging, and $P_{x y}$ is the off-diagonal component of stress tensor given by:

$$
P_{x y}=\sum_{i=1}^{N} m_{i} v_{i}^{x} v_{i}^{y}-\sum_{i=1}^{N} \sum_{j>i}^{N} r_{i j}^{x} \frac{\partial U_{i j}}{\partial r_{i j}^{y}}
$$

where $N, m$, and $v$ are, respectively, the total number, mass, and velocity of liquid molecules $i$ and $j$.

Our calculated viscosity for water TIP3P model is $0.31 \pm 0.08 \mathrm{mPa} \mathrm{s}$, which is in good agreement with MD study of González and Abascal [41], and for decane is $0.63 \pm 0.06 \mathrm{mPa} \mathrm{s}$. Considering the radius of $1.5 \mathrm{~nm}$, diffusion coefficients of $4.57 \times 10^{-6} \mathrm{~cm}^{2} / \mathrm{s}$ and $2.32 \times 10^{-6} \mathrm{~cm}^{2} / \mathrm{s}$ are obtained using the SE relation for a spherical nanoparticle in water and decane, respectively.

Diffusion coefficients for NPs obtained with the aid of Equation (4) are presented in Table 7. The diffusion coefficients of all the NPs, except HSNP and FSNP25, in water are greater than those predicted by the SE relation. Also, the diffusion coefficients of all the NPs, except HSNP, in decane are greater than those predicted by the SE relation. Interestingly, if we consider larger effective radius for our functionalized NPs, due to their functional groups, the SE relation provides a smaller diffusion coefficient, but $\mathrm{MD}$ results completely negate this prediction. High surface activity of fluorinated NPs reduces residence time of liquid molecules and extent of their binding to the NPs, and eases the diffusion of fluorinated NPs across water or decane. The results indicate that diffusion coefficient of NPs increases with increasing the number of water and oil repellent groups on the nanoparticle's surfaces. The simulated diffusion coefficients suggest that both of greater numbers and larger fluorocarbon chains on the NPs eases diffusion of functionalized NPs across the bulk liquid.

\subsection{Potential of Mean Force Calculations}

An imperative requirement for the suitability of wettabilitymodifier chemicals is that they must adsorb on the mineral surface and not aggregate in clusters. To achieve this goal, it is preferred that the free energy of aggregation for two similar NPs be less negative than the free energy of adsorption for one of them [42]. Consequently, PMF calculations were conducted to determine the free energy of adsorption of NPs on the hydroxylated $\alpha$-quartz 001 surface and the free energy of aggregation of two similar NPs in both water and decane.

To perform PMF calculations, we constrained the distance between the surface oxygen atoms of $\alpha$-quartz slab and surface hydrogen atoms of a given nanoparticle at 40 different positions starting from $1 \AA$ with $0.5 \AA$ steps using the freeze subroutine of DL_POLY_CLASSIC. At each position, empty space of the simulation cell was filled with $1 \mathrm{~g} \mathrm{~cm}^{-1}$ water or $0.73 \mathrm{~g} \mathrm{~cm}^{-1}$ decane and a $1 \mathrm{~ns}$ MD simulation was run. The adsorption free energy was then calculated by integrating the average force, $f_{z}$, required to 
restrain the nanoparticle at a specific distance, $z_{0}$, in the direction upright to the surface of $\alpha$-quartz slab [43, 44]:

$$
\Delta G\left(z_{0}\right)=\int_{0}^{z_{0}}\left\langle f_{z}(z)\right\rangle \mathrm{d} z
$$

Obtaining free energy of aggregation for two similar NPs was done in the same manner as free energy of adsorption. One of the NPs was fixed at a specific position and the other one was positioned at different distances from the fixed one both in water and decane. The separation distance is defined by the distance between the surface hydrogen atoms of NPs.

In our previous work [22], PMF calculations for the first set of our NPs were presented and discussed. Here we only present PMF data for the second set of the NPs. The free energy profile for adsorption of the second set of the NPs on hydroxylated $\alpha$-quartz surface and aggregation of them in water or decane-loaded pores are presented in Figures 7 and 8. For each of the NPs, adsorption and aggregation profiles show a global minima at the interval $(3,6) \AA$, where the corresponding value is the free energy of adsorption and aggregation. For all of the NPs in both water and decane-loaded pores, the absolute value of aggregation free energy is found to be less than the absolute value of adsorption free energy, which is required for the NPs to adsorb preferably on the hydroxylated $\alpha$-quartz surface. In addition, the free energy of adsorption becomes more favorable with increasing the number of $-\mathrm{CF}_{2} \mathrm{CF}_{3}$ groups on the NPs.

Another information that free energy profiles provide is the amount of energy needed for NPs to be adsorbed on the mineral surface or another nanoparticle. Interestingly, the energy barrier for the adsorption of the second set of our NPs on the mineral surface is zero. This result indicates that the fluorinated NPs require no work to compete with the adsorbed water or decane molecules when approaching the mineral surface.

PMF calculations done in this work is a preliminary attempt to estimate the real rate of aggregation and adsorption of NPs when mineral surface is present. These free energies can be used to estimate the ratio of NPs adsorbed on the surface to that aggregated in clusters as $K_{\mathrm{ads}} / \mathrm{K}_{\mathrm{agg}}$, as $K_{\text {ads }}=\exp \left(-\Delta G_{\text {ads }} / R T\right)$ and $K_{\text {agg }}=\exp \left(-\Delta G_{\text {agg }} / R T\right)$ are the adsorption and aggregation constants, respectively. Calculations show that $K_{\text {ads }} / K_{\text {agg }}$ is a very big number in $300 \mathrm{~K}$, suggesting that, when mineral surface is present, NPs will preferably adsorb. In real case the aggregation would contain more than two NPs, as the result adsorption free energy per particle in such a cluster would probably be lower. It is also possible, that NPs will first aggregate and only then go to the surface. These possibilities should be analyzed and discussed in future works.
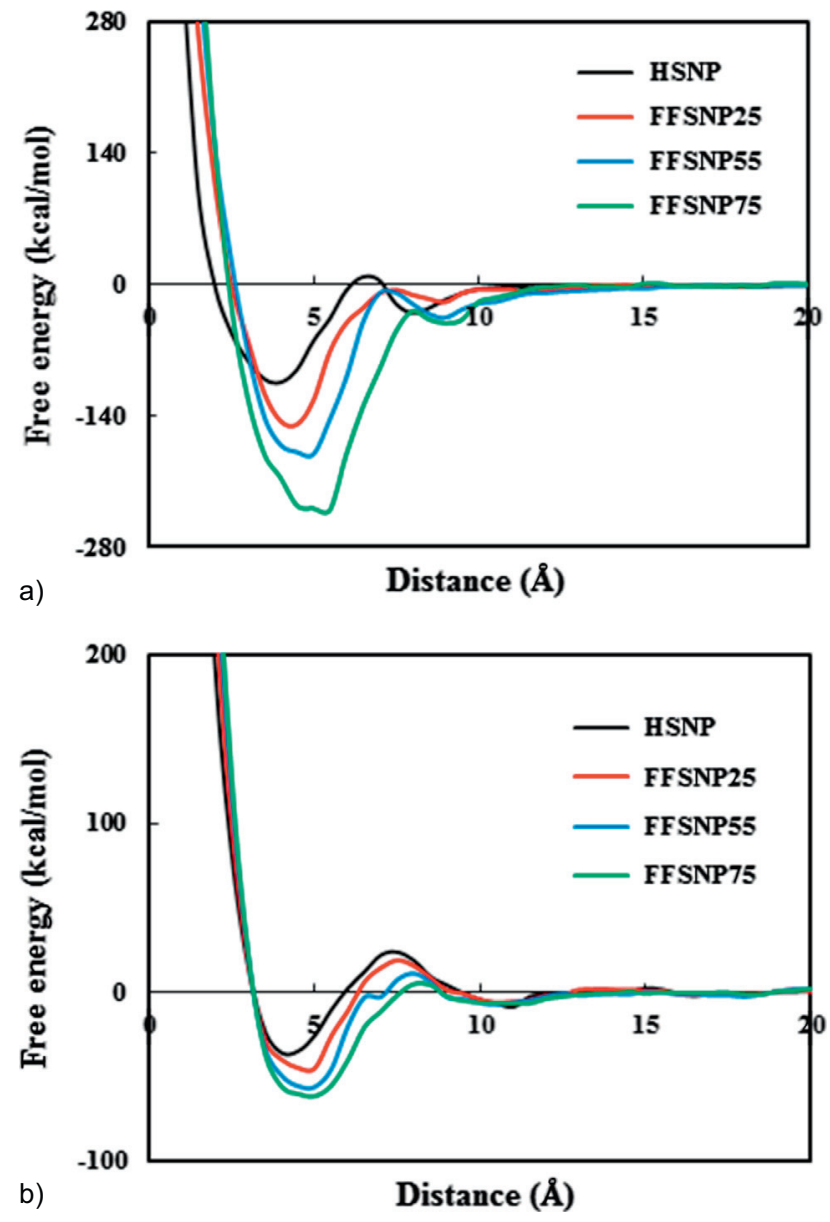

Figure 8

The free energy calculated for the NPs functionalized with $\mathrm{CF}_{2} \mathrm{CF}_{3}$ groups in a decane-loaded pore. $(\mathrm{a}, \mathrm{b})$ The free energy of adsorption on hydroxylated $\alpha$-quartz surface and the free energy of aggregation for the NPs.

\section{CONCLUSIONS}

We have applied MD simulations to examine the impact of the surface chemistry of silica NPs on their surface and transport properties in unbounded water or decane. The nanoparticle's surface chemistry was altered by varying their surface groups. Trifluoromethyl or pentafluoroethyl groups were used as hydro- and oleo-phobic sites. As the number or length of the fluorocarbon groups on the NPs' surfaces increases, density of liquid molecules in the solvation shells of the NPs decreases. It was observed that liquid-density fluctuations become larger with increasing the number of fluorocarbon groups on the NPs. The same effect was observed when the length of the groups increased. Also, residence time of liquid molecules in the first hydration shell of the NPs decreases when the number or the length of the groups increases. This effect results 
in weak binding of liquid molecules to the NPs and eases diffusion of them across the liquids. We found that the Stokes-Einstein relation fails to predict diffusion coefficient of functionalized NPs. Finally, potential of mean force calculations were undertaken, demonstrating that the NPs adsorb preferably on the hydroxylated $\alpha$-quartz 001 surface and not aggregate in the clusters. Increasing the number and the length of the fluorocarbon groups on the silica NPs makes them more appropriate for enhancement of gas recovery.

\section{ACKNOWLEDGMENTS}

I would like to thank Mr. Reza Alipour for translating title and abstract into French and for his helpful comments on earlier versions of this article. I also thank Dr. Aliasghar Mohammadi for his critical remarks and advice, and for carefully reading this manuscript.

\section{REFERENCES}

1 Prodanovic M., Ryoo S., Rahmani A.R., Kuranov R.V., Kotsmar C., Milner T.E., Johnston K.P., Bryant S.L., Huh C. (2010) Effects of magnetic field on the motion of multiphase fluids containing paramagnetic nanoparticles in porous media, SPE Improved Oil Recovery Symposium, 24-28 April, Tulsa, Oklahoma, USA, SPE-129850-MS.

2 Cai J., Chenevert M.E., Sharma M.M., Friedheim J.E. (2012) Decreasing water invasion into Atoka shale using nonmodified silica nanoparticles, SPE Drill. Completion. 27, 01, 103-112.

3 Zhang T., Davidson D., Bryant S.L., Huh C. (2010) Nanoparticle-stabilized emulsions for applications in enhanced oil recovery, SPE Improved Oil Recovery Symposium, 24-28 April, Tulsa, Oklahoma, USA, SPE-129885-MS.

4 Metin C.O., Baran J.R. Jr., Nguyen Q.P. (2012) Adsorption of surface functionalized silica nanoparticles onto mineral surfaces and decane/water interface, J. Nanopart. Res. 14, 11, $1-16$.

5 Nazari Moghaddam R., Bahramian A., Fakhroueian Z., Karimi A., Arya S. (2015) Comparative study of using nanoparticles for enhanced oil recovery: Wettability alteration of carbonate rocks, Energy Fuels 29, 4, 2111-2119.

6 Kaasa A.T. (2013) Investigation of how silica nanoparticle adsorption affects wettability in water-wet Berea Sandstone, Master Thesis, NTNU, Trondheim, Norway.

7 Mousavi M., Hassanajili S., Rahimpour M. (2013) Synthesis of fluorinated nano-silica and its application in wettability alteration near-wellbore region in gas condensate reservoirs, Appl. Surf. Sci. 273, 205-214.

8 Kewen L., Abbas F. (2000) Experimental study of wettability alteration to preferential gas-wetting in porous media and its effects, SPE Reserv. Eval. Eng. 3, 02, 139-149.

9 Jamadagni S.N., Godawat R., Garde S. (2011) Hydrophobicity of proteins and interfaces: Insights from density fluctuations, Annu. Rev. Chem. Biomol. Eng. 2, 147-171.
10 Accordino S.R., de Oca J.M.M., Fris J.A.R., Appignanesi G.A. (2015) Hydrophilic behavior of graphene and graphene-based materials, J. Chem. Phys. 143, 15, 154704.

11 Stukan M.R., Ligneul P., Boek E.S. (2012) Molecular dynamics simulation of spontaneous imbibition in nanopores and recovery of asphaltenic crude oils using surfactants for EOR applications, Oil Gas Sci. Technol. - Rev. IFP 67, 5, 737-742.

12 Bang V. (2007) Development of a successful chemical treatment for gas wells with condensate or water blocking damage, ProQuest, Michigan, USA.

13 Koparde V.N., Cummings P.T. (2007) Molecular dynamics study of water adsorption on $\mathrm{TiO}_{2}$ nanoparticles, J. Phys. Chem. C 111, 19, 6920-6926.

14 Fan H., Resasco D.E., Striolo A. (2011) Amphiphilic silica nanoparticles at the decane-water interface: insights from atomistic simulations, Langmuir 27, 9, 5264-5274.

15 Rudyak V.Y., Krasnolutskii S.L., Ivanov D.A. (2011) Molecular dynamics simulation of nanoparticle diffusion in dense fluids, Microfluidics Nanofluidics 11, 4, 501-506.

16 Gao W., Jiao Y., Dai L.L. (2016) The effects of size, shape, and surface composition on the diffusive behaviors of nanoparticles at/across water-oil interfaces via molecular dynamics simulations, J. Nanopart. Res. 18, 4, 1-11.

17 Li Z. (2009) Critical particle size where the Stokes-Einstein relation breaks down, Phys. Rev. E 80, 6, 061204.

18 Grabowski C.A., Mukhopadhyay A. (2014) Size effect of nanoparticle diffusion in a polymer melt, Macromolecules 47, 20, 7238-7242.

19 Eslami H., Rahimi M., Müller-Plathe F. (2013) Molecular dynamics simulation of a silica nanoparticle in oligomeric poly (methyl methacrylate): A model system for studying the interphase thickness in a polymer-nanocomposite via different properties, Macromolecules 46, 21, 8680-8692.

20 Litton D.A., Garofalini S.H. (2001) Modeling of hydrophilic wafer bonding by molecular dynamics simulations, J. Appl. Phys. 89, 11, 6013-6023.

21 Makimura D., Metin C., Kabashima T., Matsuoka T., Nguyen Q., Miranda C.R. (2010) Combined modeling and experimental studies of hydroxylated silica nanoparticles, J. Mater. Sci. 45, 18, 5084-5088.

22 Sepehrinia K., Mohammadi A. (2016) Wettability alteration properties of fluorinated silica nanoparticles in liquidloaded pores: An atomistic simulation, Appl. Surf. Sci. 371, 349-359.

23 Youngs T. (2010) Aten - An application for the creation, editing, and visualization of coordinates for glasses, liquids, crystals, and molecules, J. Comput. Chem. 31, 3, 639-648.

24 Smith W., Forester T. (1996) DL POLY 2.0: A generalpurpose parallel molecular dynamics simulation package, J. Mol. Graph. Model. 14, 3, 136-141.

25 Lopes P.E., Murashov V., Tazi M., Demchuk E., MacKerell A.D. (2006) Development of an empirical force field for silica. Application to the quartz-water interface, J. Phys. Chem. B 110, 6, 2782-2792.

26 Notman R., Walsh T.R. (2009) Molecular dynamics studies of the interactions of water and amino acid analogues with quartz surfaces, Langmuir 25, 3, 1638-1644.

27 Chilukoti H.K., Kikugawa G., Ohara T. (2014) Structure and transport properties of liquid alkanes in the vicinity of $\alpha$-quartz surfaces, Int. J. Heat Mass Transfer 79, 846-857. 
28 Jorgensen W.L., Chandrasekhar J., Madura J.D., Impey R.W., Klein M.L. (1983) Comparison of simple potential functions for simulating liquid water, J. Chem. Phys. 79, 2 , 926-935.

29 Vanommeslaeghe K., MacKerell A.D. Jr. (2012) Automation of the CHARMM General Force Field (CGenFF) I: Bond perception and atom typing, J. Chem. Inf. Model. 52, 12, 3144-3154.

30 Vanommeslaeghe K., Raman E.P., MacKerell A.D. Jr. (2012) Automation of the CHARMM General Force Field (CGenFF) II: Assignment of bonded parameters and partial atomic charges, J. Chem. Inf. Model. 52, 12, 3155-3168.

31 Vanommeslaeghe K., Hatcher E., Acharya C., Kundu S., Zhong S., Shim J., Darian E., Guvench O., Lopes P., Vorobyov I. (2010) CHARMM general force field: A force field for drug-like molecules compatible with the CHARMM all-atom additive biological force fields, J. Comput. Chem. 31, 4, 671-690.

32 Leroch S., Wendland M. (2013) Influence of capillary bridge formation onto the silica nanoparticle interaction studied by grand canonical Monte Carlo simulations, Langmuir 29, 40, 12410-12420.

33 Wang J., Kalinichev A.G., Kirkpatrick R.J. (2006) Effects of substrate structure and composition on the structure, dynamics, and energetics of water at mineral surfaces: A molecular dynamics modeling study, Geochim. Cosmochim. Acta 70, 3, 562-582.

34 Li Y., Jiang G., Li L., Xu W., Yu Y., Zhang X., Xie S. (2014) The effect of a novel gas-wetting reversal FC-1 on the condensate gas reservoir core, Pet. Sci. Technol. 32, 1, 1-7.

35 Sharifzadeh S., Hassanajili S., Rahimpour M. (2013) Wettability alteration of gas condensate reservoir rocks to gas wetness by sol-gel process using fluoroalkylsilane, J. Appl. Polym. Sci. 128, 6, 4077-4085.
36 Acharya H., Vembanur S., Jamadagni S.N., Garde S. (2010) Mapping hydrophobicity at the nanoscale: Applications to heterogeneous surfaces and proteins, Faraday Discuss. 146, 353-365.

37 Impey R., Madden P., McDonald I. (1983) Hydration and mobility of ions in solution, J. Phys. Chem. 87, 25, 5071-5083.

38 Cheung D.L. (2010) Molecular simulation of nanoparticle diffusion at fluid interfaces, Chem. Phys. Lett. 495, 1, 55-59.

39 Rudyak V.Y., Belkin A., Ivanov D.A., Egorov V.V. (2008) The simulation of transport processes using the method of molecular dynamics. Self-diffusion coefficient, High Temp. 46, 1, 30-39.

40 Allen M.P., Tildesley D.J. (1987) Computer simulation of liquids, Oxford University Press, Oxford, United Kingdom.

41 González M.A., Abascal J.L. (2010) The shear viscosity of rigid water models, J. Chem. Phys. 132, 9, 096101.

42 Zhu R., Molinari M., Shapley T.V., Parker S.C. (2013) Modeling the interaction of nanoparticles with mineral surfaces: Adsorbed C60 on pyrophyllite, J. Phys. Chem. A 117, 30, 6602-6611.

43 Kerisit S., Cooke D.J., Spagnoli D., Parker S.C. (2005) Molecular dynamics simulations of the interactions between water and inorganic solids, J. Mater. Chem. 15, 14, 1454-1462.

44 Kerisit S., Parker S.C. (2004) Free energy of adsorption of water and metal ions on the 1014 calcite surface, J. Am. Chem. Soc. 126, 32, 10152-10161.

Manuscript submitted in October 2016

Manuscript accepted in April 2017

Published online in June 2017

Cite this article as: K. Sepehrinia (2017). Molecular Dynamics Simulation for Surface and Transport Properties of Fluorinated Silica Nanoparticles in Water or Decane: Application to Gas Recovery Enhancement, Oil Gas Sci. Technol 72, 17. 